\title{
Preparation and Characterization of Diblock Copolymers of the AB and CD Types and their Self-Assembled Structure by Hydrogen Bonding Interaction
}

\author{
Takeshi Asari, Shigeo Matsuo, Atsushi Takano, and Yushu Matsushita ${ }^{\dagger}$ \\ Department of Applied Chemistry, Graduate School of Engineering, Nagoya University, \\ Furo-cho, Chikusa-ku, Nagoya 464-8603, Japan
}

(Received September 12, 2005; Accepted October 27, 2005; Published March 15, 2006)

\begin{abstract}
A pair of diblock copolymers, poly(isoprene-block-2-vinylpyridine) (IP) and poly(styrene-block-(4tert-butoxystyrene)) (StB) were successfully prepared by controlled anionic polymerizations. StB diblock copolymer was treated with $\mathrm{HCl}$ and hence poly(4-tert-butoxystyrene) can be converted into poly(4-hydroxylstyrene) $(\mathrm{H})$ by an acid hydrolysis reaction, where conversion yield was $98 \%$, so that poly(styrene-block-4-hydroxystyrene) (SH) was produced quantitatively. I/P and S/H volume ratios were both designed to be 9/1. From the comparison of IR spectra of three IP/SH blends with composition ratio of $25 / 75,50 / 50$ and $75 / 25$ at the bulk film state, the formation of hydrogen bonding between a hydroxyl group on $\mathrm{H}$ and a nitrogen atom on $\mathrm{P}$ was confirmed, whose magnitude reaches to a maximum when IP/SH blend ratio is about $50 / 50$ by weight. From morphological observation using transmission electron microscopy (TEM), macrophase-separated structure was observed for IP $/ \mathrm{StB}=50 / 50$ blend, while simple lamellar microphase-separated structure was observed for IP/SH $=50 / 50$ blend due to hydrogen bonding interaction.

[DOI 10.1295/polymj.38.258]

KEY WORDS Block Copolymer Blends / Microphase-separated Structure / Hydrogen Bonding / Interpolymer Association /
\end{abstract}

In general, two different polymers are incompatible in many cases, since polymer blends are energetically unfavorable to form a simple phase due to a little gain of entropy of mixing. However some compatible polymer blends, such as polystyrene/poly(vinylmethylether), ${ }^{1}$ polystyrene/poly(2,6-dimethylphenyleneoxide) ${ }^{2}$ and poly(4-trimethylsilylstyrene)/polyisoprene ${ }^{3}$ have been already discovered. In recent years several attempts have been made to design a new category of polymer blends by introducing various strong chemical interaction into blends, including ion-ion pair interaction, ${ }^{4-6}$ hydrogen bonding, ${ }^{5,6}$ charge transfer interaction $^{7}$ etc., where energetic gain from the enthalpy of mixing allows the components to form the mixed state.

In early 1980s, several types of approaches were reported. Eisenberg et al. used the cationic and anionic groups for miscible polymer pairs, ${ }^{8,9}$ and Percec et al. reported on the miscibility enhancement by the interaction between the electron-donor and electron-acceptor attached on different polymer chains. ${ }^{7}$ As for the hydrogen bonding interaction, Pearce and Kwei systematically studied blends of poly(styrene-co-4-hydroxystyrene) with hydroxyl group and a counter polymer having carbonyl group. ${ }^{10,11}$ In these studies miscible blends were formed from some immiscible polymer pairs by introducing several attractive interactions. Among these interactions, hydrogen bonding interaction $^{12}$ must be most fascinating, since they quite efficiently vary the miscibility of polymer pairs without changing the physical properties of the main component polymers much. ${ }^{13}$

Dai et al. reported on poly(2-vinylpyridine) and poly(4-hydroxystyrene) blend system as a compatible polymer blend having hydrogen bonding interaction. ${ }^{14}$ It has been clarified that hydrogen bonding between a nitrogen atom on a pyridine ring and a phenolic hydroxyl group leads to a miscible blend by IR and DSC measurements. In their system, strong multiple hydrogen bondings lead to interpolymer complexations, therefore, 1:1 interpolymer complex composed of poly(2-vinylpyridine) and poly(4-hydroxystyrene) are obtained in the form of coprecipitate from the solution of ethanol, although it is a common good solvent. If a pair of $A B$ and $C D$ copolymers are blended in a good solvent for four component polymers, where $\mathrm{B}$ and $\mathrm{D}$ components are interactive via hydrogen bonding, a large scale miceller aggregates will be formed in solution. ${ }^{15}$ Hogen-Esch et al. reported on such a association-dissociation behavior dynamics of $\mathrm{AB} / \mathrm{CD}$ diblock copolymer blends, ${ }^{16}$ where $\mathrm{B}$ and $\mathrm{D}$ components are attractive via hydrogen bonding interaction each other, however, the complex structures were observed merely in the solution state. Furthermore very characteristic microphase-separated structures were found from the blends of $\mathrm{ABC}$ linear tri-

${ }^{\dagger}$ To whom correspondence should be addressed (Tel: +81-52-789-4604, Fax: +81-52-789-3210, E-mail: yushu@apchem.nagoya-u.ac.jp). 
block and DA diblock copolymers by Abetz et al., where $\mathrm{C}$ and $\mathrm{D}$ are miscible via hydrogen bonding so that these polymer blends can be mixed into one phase. ${ }^{17}$

Against the background of these experimental facts, we compare the bulk morphologies of two types of $\mathrm{AB} / \mathrm{CD}$ and $\mathrm{AB} / \mathrm{CD}^{\prime}$ diblock copolymer blends, one is just a simple copolymer blend without particular interaction between component polymers, while the other includes two attractive chemical components B and $\mathrm{D}^{\prime}$ through hydrogen bonding. Polyisoprene (I) and polystyrene $(\mathrm{S})$ were chosen as polymer species $\mathrm{A}$ and $\mathrm{C}$ in this work. Poly(2-vinylpyridine) (P) and poly(4-hydroxystyrene) $(\mathrm{H})$ are good candidates for $\mathrm{B}$ and $\mathrm{D}^{\prime}$ polymer components since $\mathrm{I}$ and $\mathrm{S}$ are immiscible with $\mathrm{P}$ and $\mathrm{H}$, therefore, these polymers are used in the present study.

Poly(4-hydroxystyrene) can be easily prepared by anionic polymerization of 4-tert-butoxystyrene, where poly(4-tert-butoxystyrene) is regarded as the component $\mathrm{D}$, followed by hydrolysis by acid treatment. ${ }^{18}$ This procedure is applicable to prepare a block copolymer system consisted of poly(4-tert-butoxystyrene) (tB) as one component block. Applying this method, we prepared a pair of diblock copolymers, i.e., poly(isoprene-block-2-vinylpyridine) (IP) and poly(styrene-block-4-hydroxystyrene) ( $\mathrm{SH}$ ) having the compositions, $\mathrm{I} / \mathrm{P}=9 / 1$ and $\mathrm{S} / \mathrm{H}=9 / 1$ to observe phase structure under the presence of hydrogen bonding interaction between these polymer pairs. If such copolymer molecules are obtained as designed, 2vinylpyridine units and 4-hydroxystyrene units can be attracted each other by hydrogen bondings stoichiometrically as schematically shown in Figure 1, so that $\mathrm{P}$ chains and $\mathrm{H}$ chains can be mixed to form one phase

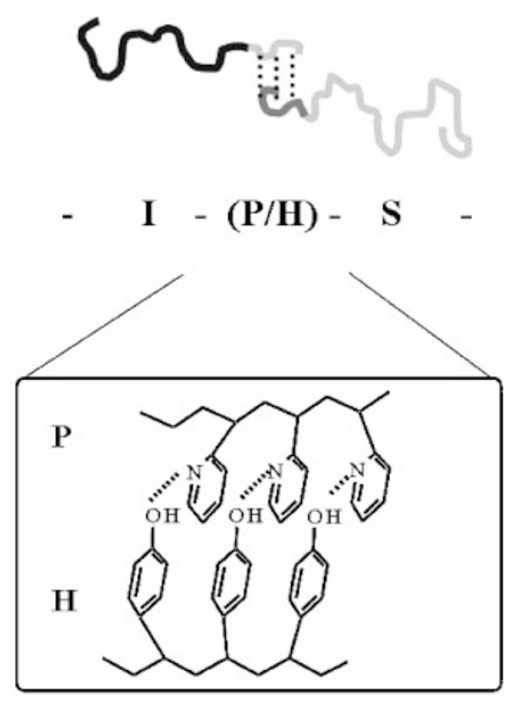

Figure 1. Schematic representation of forming hydrogen bonding between pyridine groups and hydroxyl groups for IP/ SH block copolymer blend in bulk. through interpolymer association even in bulk. This associated block copolymer with hydrogen bonding might produce a new type of block copolymer molphology. The effect of hydrogen bonding on phase structure may become clear if we compare the results for IP/SH blend with that of IP/StB blend, which does not have interacting species. In this paper, preparation and characterization of parent diblock copolymers and phase structures from diblock copolymer blends are reported.

\section{EXPERIMENTAL SECTION}

\section{Preparation of Polymer Samples}

Monodisperse block copolymer samples, that is, poly(isoprene-block-2-vinylpyridine) (IP) and poly(styrene-block-4-tert-butoxystyrene) (StB) were separately prepared on the basis of two-step living anionic polymerizations. IP diblock copolymer was synthesized in THF using sec-BuLi as an initiator at $-78^{\circ} \mathrm{C}$, where the composition, i.e., $\mathrm{I} / \mathrm{P}=9 / 1$, was designed. Microstructure of polyisoprene sequences was measured by ${ }^{1} \mathrm{H}$ NMR. A StB diblock copolymer with the composition of $\mathrm{S} / \mathrm{tB}=9 / 1$, was synthesized similarly under almost the same experimental conditions. Hydrolysis of tert-butoxy group was carried out under acidic condition with $\mathrm{HCl}$. Dried StB was dissolved into 1,4-dioxane (5 wt \% solution) and kept at $40^{\circ} \mathrm{C}$ under $\mathrm{N}_{2}$, successibly conc. $\mathrm{HCl}$ was gradually added under $\mathrm{N}_{2}$. This solution was stirred for $24 \mathrm{~h}$ at $40^{\circ} \mathrm{C}$, followed by pouring into distilled water. Precipitate was collected by filtration through a glass filter and rinsed with distilled water until complete neutralization was attained with $\mathrm{PH}$ at 7 . Collected precipitate was dissolved into 1,4-dioxane, dust was excluded by filtration, successively polymer was obtained by freeze-drying from 1,4-dioxane solution. Degree of hydrolysis for the $\mathrm{SH}$ diblock copolymer was determined by ${ }^{1} \mathrm{H}$ NMR. Thus SH copolymer with designed composition, $\mathrm{S} / \mathrm{H}=9 / 1$, was also prepared.

\section{Molecular Characterization of Polymers and Prepa- ration of Blend Films}

Molecular weights of polyisoprene and polystyrene precursors were measured by a multi angle laser light scattering apparatus of Wyatt Technology in THF at $35^{\circ} \mathrm{C}$, model DOWN-EOS. The $\mathrm{dn} / \mathrm{dc}$ values estimated are 0.185 for polystyrene and 0.126 for polyisoprene in THF at $35^{\circ} \mathrm{C}$ by interferometric reflectmeter, OPTILAB DSP, of Wyatt Technology. Molecular weight distribution was estimated by gel permeation chromatography using HLC-8020 of TOSOH Co. with THF as an eluent at $38^{\circ} \mathrm{C}$ equipped with three $\mathrm{G} 4000 \mathrm{H}_{\mathrm{HR}}$ columns of TOSOH. About $2 \mathrm{wt} \%$ tetramethylethylenediamine was added to THF to avoid 
adsorption of poly(2-vinylpyridine) block on polystyrene gel. ${ }^{1} \mathrm{H}$ NMR analysis was used to determine the composition of the block copolymers. Solvents used were $\mathrm{CDCl}_{3}$ for StB and IP, mixed solvent of $\mathrm{CDCl}_{3} /\left(\mathrm{CD}_{3}\right)_{2} \mathrm{SO}$ for $\mathrm{SH}$.

For the film preparation, $5 \mathrm{wt} \%$ THF stock solutions of SH and IP diblock copolymers were separately prepared. Blend thin films were made by solvent casting from the mixture of these solutions. Appropriate amounts of these solutions were added to the teflon petri dishes and evaporated for two days, followed by drying for another two days in a vacuum oven. Three different blends were prepared, their IP/SH ratio are $25 / 75,50 / 50,75 / 25$ by weight. IP/StB blend thin film with relative ratio of 50/50 was made using similar method. Dried sample films were transparent for $\mathrm{IP} / \mathrm{SH}$, slightly turbid for IP/StB, and they were used for measuring FT/IR spectrum by JASCO, model FT/IR-620 to observe the hydrogen bonding. The glass transition temperature $\left(T_{\mathrm{g}}\right)$ was measured with differential scanning calorimetry (DSC) using a EXSTAR6100 DSC of Seiko Instruments Inc. The measurements were performed at a heating rate of $10^{\circ} \mathrm{C} /$ min after heating for $1 \mathrm{~h}$ and quenching to $-50^{\circ} \mathrm{C}$ under a nitrogen atmosphere. $T_{\mathrm{g}}$ values were estimated from the midpoint of the heat capacity jump. Obtained blend sample films were cut into ultrathin sections with thickness of $c a .50 \mathrm{~nm}$ by an ultramicrotome, Ultracut UCT of LEICA. The sections were stained with $\mathrm{OsO}_{4}$ for $8 \mathrm{~h}$ and their microphase-separated structures were observed by a transmission electron microscope (TEM), H-800, of Hitachi under an acceleration voltage of $100 \mathrm{kV}$. In the TEM image, it is known from our experience for several simple cases that I, P, H, tB and S show deep, intermediate, light, more light and very light contrasts, respectively, by $\mathrm{OsO}_{4}$ staining.

\section{RESULTS AND DISCUSSION}

Figure 2 shows the GPC chromatograms of IP and StB diblock copolymers, together with those of the precursors. The difference in peak locations between block copolymers and the corresponding homopolymers is much smaller for IP-91, reflecting that the characteristic GPC elution time for component $\mathrm{P}$ is longer than those of the others. It is evident that two block copolymers possess unimodal and symmetric peaks showing narrow molecular weight distribution. The ${ }^{1} \mathrm{H}$ NMR spectra of three diblock copolymers are compared in Figure 3. The signals around $1.2 \mathrm{ppm}$ at the middle chart in Figure 3 are attributed to the tert-butyl groups in poly(4-tert-butoxystyrene) chain, while the same signal is hardly observed at the bottom chart for SH. This means almost all of the tert-butoxy

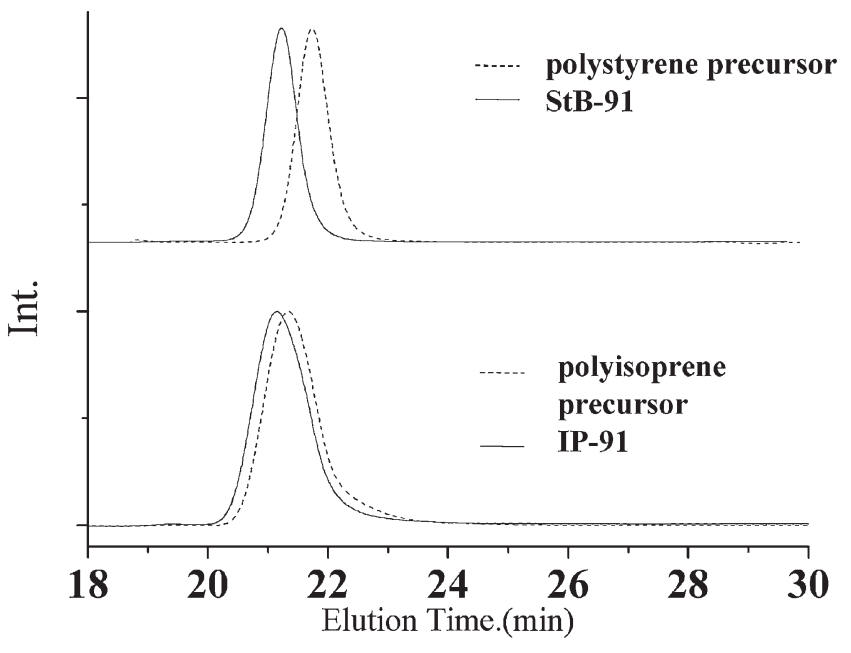

Figure 2. GPC chromatograms of two parent diblock copolymers and their precursors.

groups in StB-91 have been converted into hydroxyl groups by the present acid treatment, while a new broad peak was observed at $8.4 \mathrm{ppm}$ at the bottom chart in Figure 3. This peak comes from hydroxyl groups in $\mathrm{H}$ component. Mole fractions of each block copolymers were estimated by comparing the integration of aromatic region (6-9 ppm, without $\mathrm{OH})$ and alkyl-vinyl region $(0.5-6 \mathrm{ppm})$. From this method, the yield of hydrolysis reaction was estimated to be $98 \%$, therefore, we conclude that this hydrolysis reaction proceed very efficiently. Microstructures of polyisoprene block in IP were determined as follows; $42 \%$ $1,2,54 \% 3,4$ and $4 \% 1,4$. Table I summarizes the estimated molecular characteristics of three diblock copolymers prepared. This table also lists $M_{\mathrm{w}}$ values of the precursors and the block copolymers together with the molecular weight distribution. The $M_{\mathrm{w}}$ 's of block copolymers were calculated using measured $M_{\mathrm{w}}$ 's of precursors and mole fractions of copolymers.

Figure 4 shows the results of the thermal analysis for the 50/50 blend of IP-91/SH-91 together with those of two parent diblock copolymers. In the curve of SH-91 diblock copolymer, $T_{\mathrm{g}}$ is evident at $104^{\circ} \mathrm{C}$, which corresponds to the transition for polystyrene block. $T_{\mathrm{g}}$ from poly(4-hydroxystyrene) phase $(\sim 180$ ${ }^{\circ} \mathrm{C}$ ) was not able to be found from this thermogram, probably because mole fraction of $\mathrm{H}$ component is too small to find heat capacity jump. Similarly, IP-91 diblock copolymer shows only one $T_{\mathrm{g}}$ at $4{ }^{\circ} \mathrm{C}$ for polyisoprene block. IP-91/SH-91 blend shows two different $T_{\mathrm{g}}$ at 3 and $103^{\circ} \mathrm{C}$, which can be conceived as endotherms for phase-separated I and S phases, though precise phase structure was not fully clarified from this experiments.

Figure 5 shows the transmission IR spectra of two diblock copolymers and their blend films with three compositions. Poly(4-hydroxystyrene) is known to 


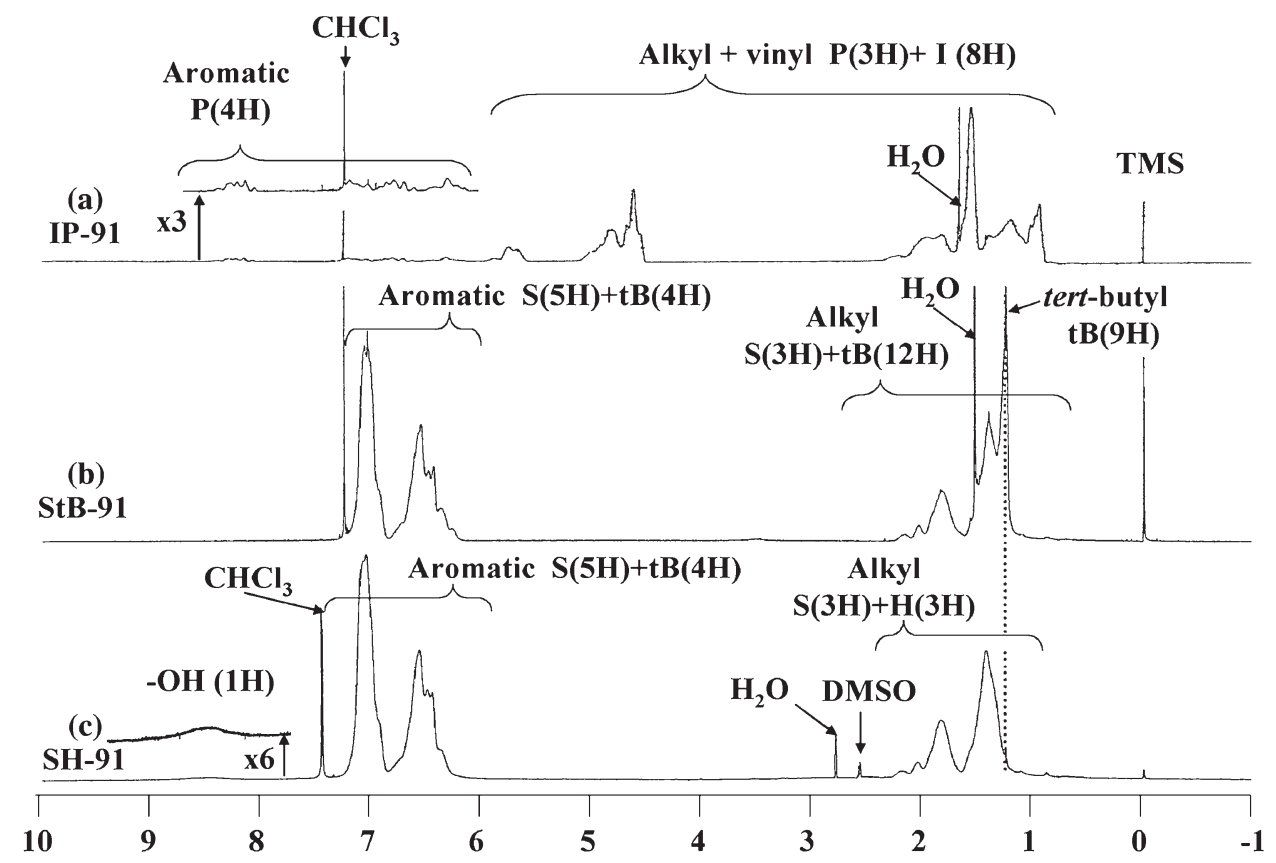

Figure 3. ${ }^{1} \mathrm{H}$ NMR spectra of (a) IP-91, (b) StB-91 and (c) the corresponding acid hydrolyzed SH-91 diblock copolymers.

Table I. Molecular characteristics of IP, StB and SH diblock copolymers

\begin{tabular}{lcccccccc}
\hline & \multicolumn{2}{c}{$M_{\mathrm{w}} \times 10^{-4}$} & & \multicolumn{4}{c}{ Mol fraction $^{\mathrm{d}}$} \\
\cline { 2 - 3 } \cline { 5 - 8 } Polymers & $\begin{array}{c}\text { Precursor } \\
\text { (S, I) }\end{array}$ & block $^{\mathrm{b}}$ & $M_{\mathrm{w}} / M_{\mathrm{n}}{ }^{\mathrm{c}}$ & & $\phi_{\mathrm{I}}$ & $\phi_{\mathrm{P}}$ & $\phi_{\mathrm{S}}$ & $\phi_{\mathrm{H}(\mathrm{StB})}$ \\
\hline IP-91 & 6.9 & 8.0 & 1.06 & 0.91 & 0.09 & - & - \\
StB-91 & 7.7 & 9.0 & 1.03 & - & - & 0.86 & 0.14 \\
SH-91 & 7.7 & 8.6 & - & - & - & 0.86 & 0.14 \\
\hline
\end{tabular}

${ }^{\mathrm{a}}$ Determined by MALLS. ${ }^{\mathrm{b}}$ Calculated from $M_{\mathrm{w}}$ of precursors and the mole fractions of block copolymers. ${ }^{\mathrm{c}}$ Estimated by GPC. Eluents are $2 \mathrm{wt} \%$ TMEDA/THF for IP, THF for $\mathrm{StB}$ and polystyrene standards. ${ }^{\mathrm{d}}$ Calculated from ${ }^{1} \mathrm{H}$ NMR spectra.

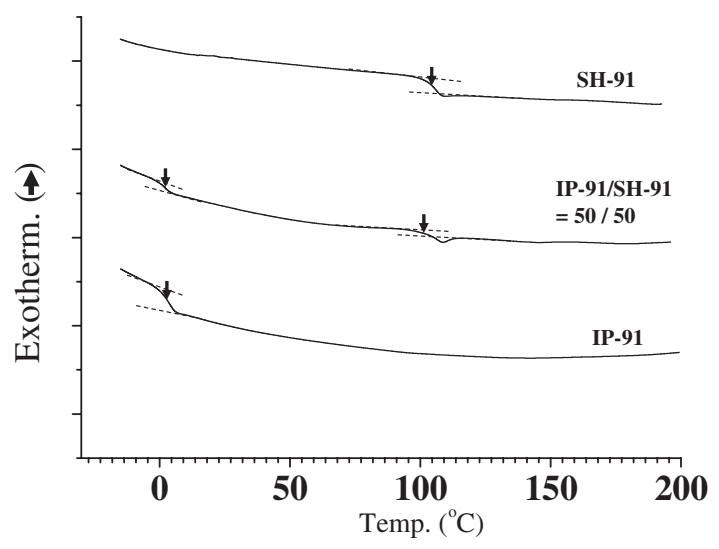

Figure 4. DSC thermogram of a IP-91/SH-91 block copolymer blend with composition of 50/50 by weight, and those of two parent block copolymers, IP-91 and SH-91.

form self-association structure through hydroxyl groups in bulk, ${ }^{13,14}$ that is, a shoulder at $3500 \mathrm{~cm}^{-1}$ for the SH diblock copolymer is characteristic to free



Figure 5. FT/IR spectra of the IP-91/SH-91 blends with various compositions together with those of parent diblock copolymers at bulk film state.

hydroxyl groups, while multiple self-associated structure between hydroxyl groups is observed at 3315 $\mathrm{cm}^{-1}$ at the bottom curve in Figure 5. Upon increasing poly(2-vinylpyridine) content of the blends from bottom to top, a peak at $3500 \mathrm{~cm}^{-1}$ disappears and the one at $3315 \mathrm{~cm}^{-1}$ shifts to lower wavelength as indicated by arrows in Figure 5. It is known for this system, this peak shift to lower wavelength can be attributed to the presence of strong interaction between a hydroxyl group and a nitrogen atom on a pyridine ring. ${ }^{14}$ This wavelength shift from hydroxyl group reaches to a maximum at the composition of 50:50, indicating that $\mathrm{P}$ and $\mathrm{H}$ block polymers are favorable to form stoicheometrically self-assembled 1:1 com- 
a)

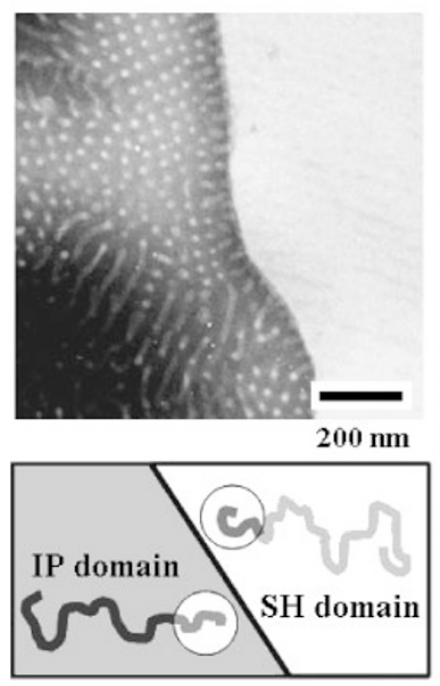

b)

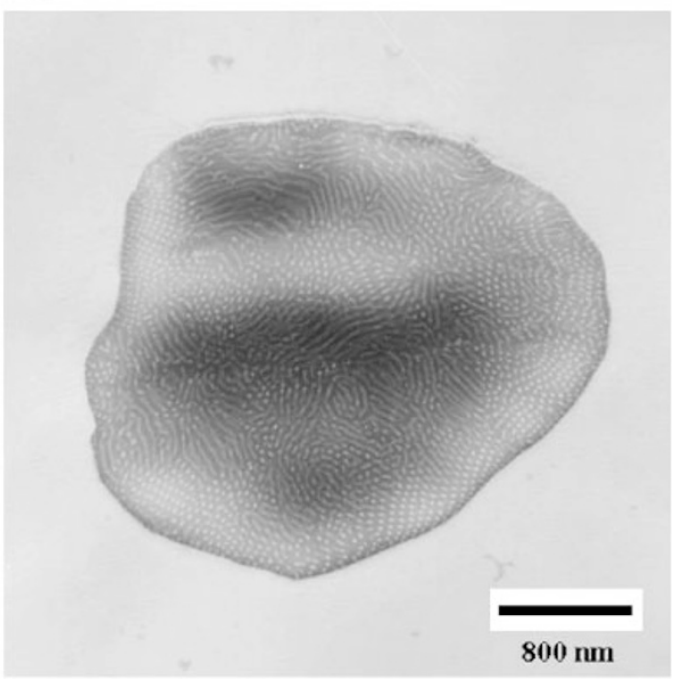

Figure 6. TEM images for an IP-91/StB-91 = 50/50 diblock copolymer blend film. (a) a typical domain boundary between two microphase-separated phases and (b) a wide-range image. Ultrathin section was stained with $\mathrm{OsO}_{4}$.

a)

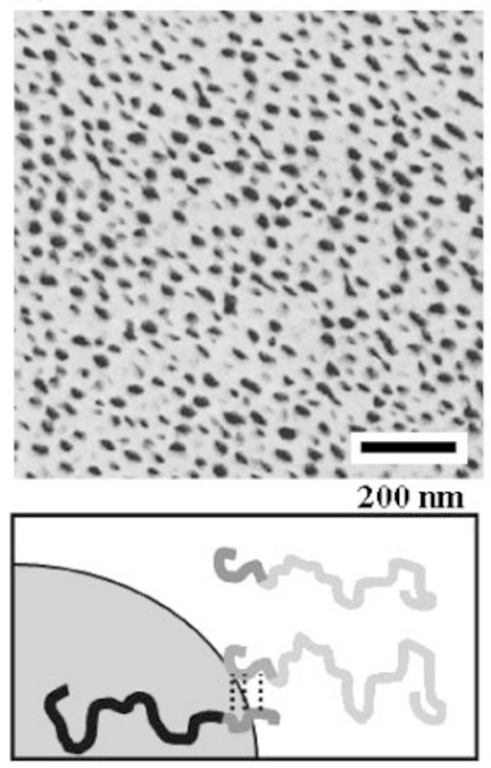

b)
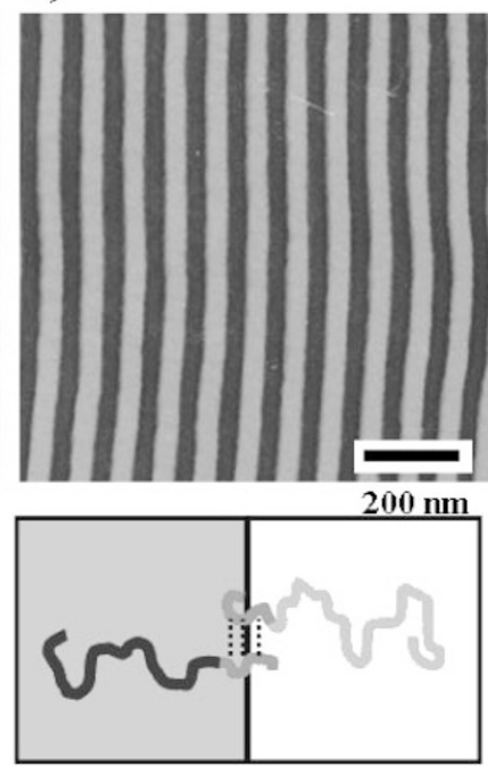

c)


Figure 7. TEM images of IP-91/SH-91 blends. Weight ratios are (a) 25/75, (b) 50/50 and (c) 75/25. Corresponding molecular arrangements is schematically shown at the bottom of each image.

plex between hydroxyl groups and pyridine groups over self association of $\mathrm{H}$ polymer. From these results, it has been found that poly(2-vinylpyridine) and poly(4-hydroxystyrene) block chains interact each other, i.e., these polymers form interpolymer complex via hydrogen bonding between these complemental chemical species.

A TEM image for the IP/StB blend is shown in Figure 6a. Macrophase separated structure between microphase separated IP (left) and StB (right) components are clearly shown. Cylindrical domain structure is recognized for the former, however, it is hard to see regular structure for the latter since the contrast between $\mathrm{S}$ and $\mathrm{tB}$ is very weak. Enlarged image is shown in Figure 6b, where macrophase separation is quite evident.

Figure 7 compares the TEM images of IP/SH blends with different compositions, i.e., (a) IP-91/ SH-91 $=25 / 75$, (b) $50 / 50$ and (c) $75 / 25$, for $\mathrm{OsO}_{4}$ stained films. In Figures $7 \mathrm{a}$ and $7 \mathrm{c}$, basically the same kind of phase-separated structures are shown, whose domain sizes are characteristic for microphase separation, though the periodicity is very low for both. In Figure 7a small dark domains of IP component co- 
polymer, which must be partially connected with $\mathrm{SH}$ by hydrogen bondings, are embedded in a matrix of excess amount of SH component, whereas small white domains of SH component are embedded in a matrix of IP as shown in Figure 7c. These assembly manners resemble those of block copolymer/homopolymer blends and they are schematically shown at the bottom of Figures $7 \mathrm{a}$ and $7 \mathrm{c}$. These heterogeneous structures were obviously caused by mismatch in molar amount between IP and SH diblock copolymers. To the contrary highly ordered periodic lamellar structure was observed for $\mathrm{IP} / \mathrm{SH}=50 / 50$ diblock copolymer blend as shown in Figure $7 \mathrm{~b}$. This structure looks very simple alternating lamellar one formed by symmetric diblock copolymer of the $\mathrm{AB}$ type.

These results indicate that hydrogen bonding interaction causes the phase transition for IP/SH diblock copolymer blends and that $\mathrm{P} / \mathrm{H}$ stoichiometric ratio is very important to form regular and homogeneous phase structure. These TEM images are consistent with the results from IR and DSC measurements. Thus very short $\mathrm{P}$ and $\mathrm{H}$ blocks play a key role to form homogeneous microphase separated structure if these two component polymers are mixed stoichiometrically, while imbalance in the amounts of $\mathrm{P}$ and $\mathrm{H}$ causes the less-ordered structure. Here we believe the structure in Figure $7 \mathrm{~b}$ is not a simple lamellar structure. Details of the structure analysis are reported elsewhere. ${ }^{19}$

\section{CONCLUSIONS}

In summary a pairs of diblock copolymer, poly(isoprene-block-2-vinylpyridine) (IP) and poly(styreneblock-4-tert-butoxystyrene) (StB) were successfully prepared by controlled anionic polymerization techniques. StB diblock copolymer is quantitatively converted into poly(styrene-block-4-hydroxystyrene) (SH) by hydrolysis with $\mathrm{HCl}$. From the IR spectrum and thermal analysis for IP/SH blends in comparison with those of parent diblock copolymers in bulk, the formation of hydrogen bonding was suggested. From morphological observation for IP/SH and IP/StB polymer blends using transmission electron microscopy, macrophase-separated structure between microphase separated StB and IP diblock copolymers was observed for the IP/StB blend, while ordered periodic and homogeneous lamellar structure was observed from $\mathrm{IP} / \mathrm{SH}=50 / 50$ due to hydrogen bonding interaction. This fact indicates stoichiometric association between $\mathrm{P}$ and $\mathrm{H}$ is favorable in bulk.
Acknowledgment. T. Asari thanks for a JSPS Research Fellowship (No. 50451) for Young Scientists. This work was also done under the support of the 21th center of excellence (COE) program entitled "The Creation of Nature-Guided Materials Processing" in the School of Engineering, Nagoya University, which has been assigned as one of the 21 st century COE programs and one of the authors Y. Matsushita is grateful to the support.

\section{REFERENCES}

1. C. C. Han, B. J. Bauer, J. C. Clark, Y. Muroga, Y. Matsushita, M. Okada, Q. Tran-cong, and T. Chang, Polymer, 29, 2002 (1988).

2. A. R. Shultz and B. M. Gendron, J. Appl. Polym. Sci., 16, 461 (1972).

3. M. Harada, T. Suzuki, M. Ohya, D. Kawaguchi, A. Takano, and Y. Matsushita, Macromolecules, 38, 1868 (2005).

4. E. Tuchida and K. Abe, Adv. Polym. Sci., 45, 1 (1982).

5. E. Becturov and L. Bimendina, Adv. Polym. Sci., 41, 99 (1981).

6. "Macromolecular Complexes in Chemistry and Biology," P. Dubin, J. Bock, R. M. Davies, D. N. Schul, and C. Thies, Ed., Springer-Verlag, Berlin, 1994.

7. J. Rodoriguez-Parada and V. Percec, Macromolecules, 19, 55 (1986).

8. A. Eisenberg, P. Smith, and Z. L. Zhow, Polym. Eng. Sci., 22, 1117 (1982).

9. Z. L. Zhou and A. Eisenberg, J. Polym. Sci. Polym. Phys. Ed., 21, 595 (1983).

10. K. J. Zhu, S. F. Chen, T. Ho, E. M. Pearce, and T. K. Kwei, Macromolecules, 23, 150 (1990).

11. L. Jong, E. M. Pearce, and T. K. Kwei, Polymer, 34, 48 (1993).

12. L. J. Prince, D. N. Reinhoudt, and P. Timmerman, Angew. Chem., Int. Ed., 40, 2382 (2001).

13. M. Jiang, M. Li, M. Xiang, and H. Zhou, Adv. Polym. Sci., 146, 121 (1999). References are there in.

14. J. Dai, S. H. Goh, S. Y. Lee, and K. S. Siow, Polym. J., 26, 905 (1994).

15. M. Xiang, M. Jiang, Y. Zhang, C. Wu, and L. Feng, Macromolecules, 30, 2313 (1997).

16. J. Pan, M. Chen, W. Warner, M. He, L. Dalton, and T. E. Hogen-Esch, Macromolecules, 33, 7835 (2000).

17. S. Jiang, A. Gopfert, and V. Abetz, Macromolecules, 36, 6171 (2003).

18. K. Se, K. Miyawaki, K. Hirahara, A. Takano, and T. Fujimoto, J. Polym. Sci., Part A: Polym. Chem., 36, 3021 (1998).

19. T. Asari, S. Matsuo, A. Takano, and Y. Matsushita, Macromolecules, 38, 8811 (2005). 